\title{
Analyzing head roll and eye torsion by means of offline image processing
}

\author{
Frédéric Sarès, Lionel Granjon, Abdelrhani Benraiss, and Philippe Boulinguez \\ Université de Poitiers, Poitiers, France
}

\begin{abstract}
Ocular torsion is a key problem in the understanding of many visual perceptual effects. However, since it is difficult to record, its integration with other sensorimotor signals is still poorly understood. Unfortunately, eyetracker systems are generally not dedicated to the monitoring of eye torsion. In addition, the classical methods used with video-based systems present some limits in the accuracy of torsion calculation. These limits are especially related to the detection of pupil center and the effects of pupil size changes. This article aims at (1) proposing a solution to analyze ocular torsion together with head roll using EyeLink II or similar equipment, (2) reviewing and adapting classical polar cross-correlation methods in order to improve the accuracy of torsion measurement, (3) providing a lower-cost method compared with the existing ones. Video sequences issued from the EyeLink II host computer monitor were recorded by means of a second computer equipped with a video acquisition card and converted into image sequences. Images were analyzed with algorithms of pupil center detection (median-based algorithm), torsion analysis (adapted polar cross-correlation method which takes into account pupil size variations) and marker tracking (head roll analysis). This method was tested on virtual eye images. Results are discussed with respect to other algorithms found in the literature.
\end{abstract}

The measurement of ocular torsion has theoretical and applied interests. For instance, the measure of ocular torsion can provide relevant clinical information to detect central (neurological) or peripheral (ophthalmological or otological) disorders (Kushner, 2004). As importantly, from a theoretical point of view, it is still unclear if the ocular torsion signal is taken into account by the central nervous system. More precisely, it is still debated if the magnitude of this movement is coded during sensorimotor transformations that occur during the switch from an eyecentered to a head-centered coordinate system. If such a degree of freedom is not integrated by the central nervous system, biases in the perception of orientation should be observed as suggested by other authors (Curthoys \& Wade, 1995; Wade \& Curthoys, 1997). Consequently, the accuracy of ocular torsion measurement is clearly of primary interest for a better understanding of visual, space and body perception.

Rotation of the eye about the line of sight is difficult to record because of its low magnitude. Since the first studies on this kind of ocular movement, several methods have been used to measure ocular torsion. The earliest ones consisted either in adjusting the orientation of a rotating bar to the bar-shape afterimages produced by a strobe flash (Donders, 1848; Finke \& Held, 1978), in gluing a straw on the eye and recording its displacement on a rotating piece of paper (Ohm, 1928), or in analyzing superimposed iris photographs (Nakayama, 1974). These methods are no longer used because they were either too subjective, too invasive, or because only an approximation of the tonic, not the phasic component of the human ocular torsion was provided. A more powerful method with good spatial and temporal resolution is the electromagnetic technique using coil lens (Collewijn, Van der Steen, Ferman, \& Jansen, 1985; Robinson, 1963). However, to avoid any coil slippage on the sclera, the lens must fit tightly to the eye. This suction increases the intraocular pressure and limits the testing time. In addition, other specific problems linked to eye-blinks generate inaccurate torsion measurement because of lens slippage (Bockisch \& Halswanter, 2001; Collewijn et al., 1985).

Promising advances in noninvasive techniques for recording of eye torsion were recently provided (e.g., laserbased eye tracker-Irie, Wilson, Jones, Bones, \& Anderson, 2002; programmable CMOS image sensors-Clarke, Ditterich, Drüen, Schönfeld, \& Steineke, 2002). Latest technical developments in digital recording devices have led to important processing improvements in sampling rate, spatial resolution and general reliability. CMOS cameras for example permit real-time image acquisition and processing (Clarke et al., 2002). However, despite these recent advances, standard video-based systems are still more extensively used. Indeed, the above mentioned studies rely on nonstandard equipment and costly methods of computing eye torsion. However, we believe that standard eye-tracking equipment can be used and classical techniques can be refined to measure ocular torsion with both an accurate and low-cost method. This article

P. Boulinguez, pbouling@univ-poitiers.fr 
proposes a solution that should allow most researchers who own standard video equipment to record easily and accurately ocular torsion by means of an offline image processing.

The difficulty of measuring ocular torsion using standard video equipment plus its small magnitude may probably be responsible for some theoretical inconsistencies observed in the literature. For example, some authors question the existence of the tonic component of the ocular torsion (e.g., Jampel \& Shi, 2002). This is the reason why it is still necessary to refine the existing tools and methods of analysis. Different algorithms have been proposed to analyze ocular torsion on the basis of video recordings: the classical polar cross correlation method (Hatamian \& Anderson, 1983), the tracking of natural landmarks of the eye (Groen, Bos, Nacken, \& De Graaf, 1996) and the template matching method (Zhu, Moore, \& Raphan, 2004). However, the first algorithm has limited precision for eccentric eye positions in the orbit. The two others require the presence of visible landmarks in the iris of the monitored eye. Moreover, all three algorithms depend on the efficiency with which the pupil center can be detected. Most of the classical methods are very simple (e.g., barycenter - center of mass - or pupil border mean), but can generate important biases due to noise, especially corneal reflection of infrared light, or image distortion related to eye eccentricity in the orbit (Hatamian \& Anderson, 1983). In addition, methods such as the polar cross-correlation necessitate the use of myotic medicines to avoid changes in the pupil size. Pilocarpine hydrochloride which is dispensed under medical prescription is often used and presents several side effects such as blurred vision, headache, and vitreous haemorrhage.

Powerful devices such as EyeLink II (SR Research Ltd., Mississauga, ON) offer an efficient compromise between spatial and temporal resolution to monitor eye movements. However, EyeLink II, like most video-based systems, is limited to the recording of two-dimensional eye movements and it is not intended to record ocular torsion. This article presents a method aimed at (1) analyzing both ocular torsion and head roll by means of the same EyeLink II equipment, (2) proposing a new simpler and more efficient method to detect pupil center, (3) adapting the algorithm to take into account the changing size of the pupil to avoid the use of myotic medicines.

\section{METHOD}

Video sequences of eye movements were recorded by means of a second computer equipped with a video acquisition card. To analyze ocular torsion, we adapted a classical polar cross-correlation-based method, setting up a new and simple median-based method for the detection of the pupil center. The method was tested with virtual (computer-generated) eye movements and illustrated with real human eye movements during head tilt.

\section{Configuration and Video Acquisition}

The system hardware is presented on Figure 1. Subjects were equipped with the EyeLink II headband (the system is head mounted, thus the recording reports eye torsion relative to the head in a manner that is unaffected by head roll, pitch, or yaw). One EyeLink II camera was used to record the subject's nondominant eye. The four EyeLink II infrared LEDs were placed in front of subject's head on the four corners of a screen facing him. The EyeLink II host computer screen displayed the image of the eye (EyeLink II setup cameras screen). In order to improve the quality of the video sequence, pupil and infrared LEDs detection (EyeLink II threshold coloring and crosshairs options) was disabled. In addition, the video output was recorded as an AVI file by means of a second computer equipped with a DV500 video acquisition card. Recordings were made using European video standard PAL (30 frames/sec, depth of 8 bits; Figure 1). It must be mentioned that there is additional loss in image resolution while recording the video output as an AVI file. The resulting resolution was $640 \times 480$ (it was $1,024 \times 768$ before digitization). The final resolution of the eye picture was $333 \times 244$ after suppression of the peripheral parts of the image that did
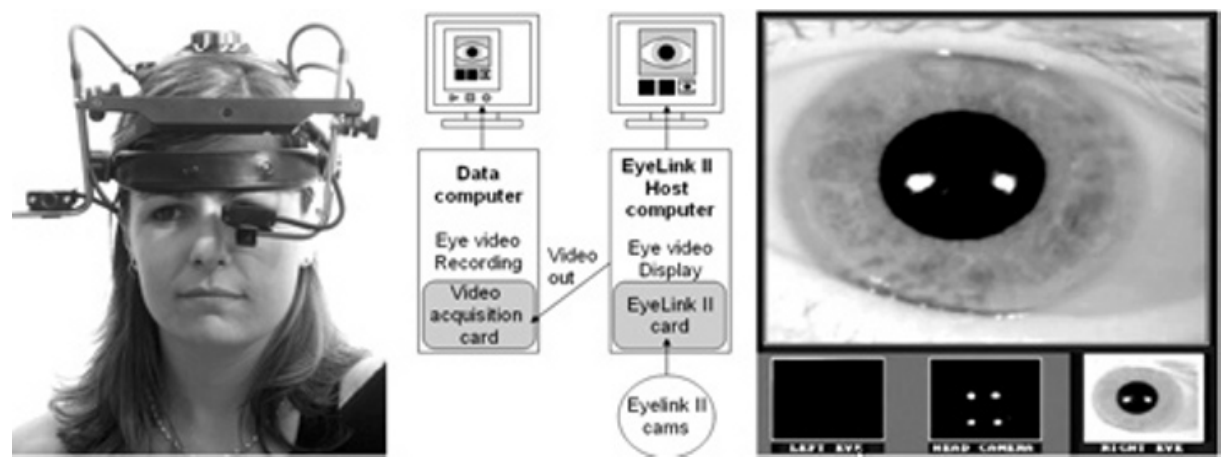

Figure 1. Illustration of the experimental setup used to record both head and eye roll with the EyeLink II system coupled with a second computer equipped with a video acquisition card. The video frame obtained with our video recording method is divided into four parts (right part of the figure): a large panel which displays the image of the filmed eye and three smaller panels. The central panel displays the four markers orientation with respect to the head. The two lateral panels display the scene filmed by the cameras. Note that the free camera can be used to record any signal that could be useful to synchronize the video sequence with any other data. 
not contain iris related information. No compression of the image was performed.

Unfortunately, there is no way of capturing the signal upstream at the source. This is not possible because the EyeLink II system must be run displaying the "camera setup screen." This mode must be used in order to have both, a large image of the eye (i.e., the best resolution for the video capture) and information about head markers. This represents the best compromise for an optimal resolution that is possible using this equipment.

The resulting frames of the video sequence were composed of one large image of the eye (a $333 \times 244$ pixel zone) and three smaller panels $(86 \times 63$ pixel zone $)$ : the central panel displayed the 4 infrared LEDs position with respect to the head by means of the EyeLink II headmounted infrared camera. The two lateral panels displayed the scene filmed by the two cameras, respectively. Note that among these latter panels, the one corresponding to the unused camera could be dedicated to the recording of a visual signal in order to synchronize the video sequence with other data. An important point is that this method does not allow to record both eyes at the same time, since the system can only display and use in the analysis one large image. Indeed, the resolution of the bottom panels is too small to obtain an accurate signal of ocular torsion. However, in normal subjects, movements of the two eyes are generally conjugated (Diamond \& Markham, 1981).

\section{Image Sequence Analysis}

An offline image processing method based on the polar cross-correlation method was used to analyze eye torsion (Hatamian \& Anderson, 1983). This method relies on the variation of intensity of an iris picture which occurs in specific directions in a polar coordinate system centered on the pupil. Head orientation was deduced from the tracking of the four markers. This method of simultaneous eye and head torsion analysis presents several steps which will be detailed in the following sections.

Pupil center tracking. The pupil is the darkest zone of the 256 gray levels image (see for illustration figure 3D). Visualization of the image histogram (frequency of occurrence of each gray level) allows definition of a pupil detection threshold since the brightness values of the iris structure generally fall between 90 and 150 in the image brightness histogram whereas the pupil occupies a range between 0 and 5 (it rarely reaches 20). A quasinull pixel density is generally observed between both. Thus, the default threshold was 40 out of 256, but a human-machine interface allows modification if necessary. All the pixels whose intensity was under this threshold were considered as a part of the pupil. An $(N \times 2)$ pupil matrix was then obtained with $N$ equals the number of pixels constituting the pupil and the two columns designating the coordinates for the abscissa and the ordinates. Since the iris zone to be analyzed is centered on the pupil, we had to determine the pupil center with accuracy. Several methods have been proposed. For instance, Hatamian and Anderson (1983) averaged the coordinates of the edge points of the pupil. The most used method relies on the determination of the "center of mass" by assigning a "mass" of 1 to each pixel constituting the pupil (Hall, 1983; Moore, Curthoys, \& McCoy, 1991; Moore, Haslwanter, Curthoys, \& Smith, 1996; Vieville \& Masse, 1987; Yamanobe, Taira, Morizono, Yagi, \& Kamio, 1990). However, these two methods have been criticized because they assume that all the pupil area remains black during the recording (Sung \& Reschke, 1997). Unfortunately, it is rarely the case because the pupil is generally partially occluded by the corneal reflection of infrared light used to light the eye. The errors induced by these methods can shift the pupil center and can lead to important errors in determining eye torsion.

To avoid such a problem, Sung and Anderson (1991) suggested fitting a circle to the perimeter of the pupil. However, this method assumes that the pupil is a perfect circle shape. This problem was addressed by ellipse fitting methods (Sung \& Reschke, 1997; Zhu, Moore, \& Raphan, 1999). The alternative method we propose here relies on a simple and efficient algorithm that takes a corrected median of the pixels constituting the pupil as the pupil center. It comprises several steps: first, the algorithm calculates two vectors, one for the abscissa and one for the ordinate (Figure 2A, the average of the points of each vector constituting the pupil in the horizontal and vertical dimensions). If no noise is provided on the pupil image, the mean value of these two vectors provides the coordinates of the pupil center. However, noise such as corneal reflection of infrared light leads to some biased values in the vector, thus leading to errors in the estimation of the pupil center. Second, the respective median was subtracted from the values of these vectors (Figure 2B). These two difference-to-median vectors were sorted after rectification (absolute value) and extreme values (beyond the 90th percentile) were rejected. Five filtering iterations were sufficient to obtain a stabilized value in the presence of strong noise upon the pupil (Figure 2B). Such a procedure constitutes a faster solution than a fitting algorithm. Finally, pupil center is deduced from the stabilized values. Such a method is straightforward, fast and very robust.

Pupil center estimation is important not only to analyze a pupil-centered iris zone (e.g., as it necessary to analyze ocular torsion), but also to track gaze direction, the two other degrees of freedom of the eye. The extraction of the vertical and horizontal angular displacements of the eye needs an accurate calibration of the system: first, the camera must film the frontal plane of the eye; second, the pupil must be centered on the video image; third the subject must gaze at targets which visual angle is previously known. In order to optimize the calibration, Schreiber and Haslwanter (2004) showed that the use of a 10-15 fixation points device may reduce the mean error below $0.2^{\circ}$. This proviso makes the conversion of the eye displacement in pixel to an angular displacement feasible. Hence, it is possible to check that eye eccentricity remains low during recording (i.e., under $10^{\circ}$ of eccentricity) in order to maintain the reliability of the polar cross-correlation method.

Transformation into polar coordinates. The iris is a muscular diaphragm that controls the size of the pupil and the amount of light that enters in the eye. On a bidimensional picture, the iris has an annulus shape whose inner diameter is constituted by the pupil diameter and whose 

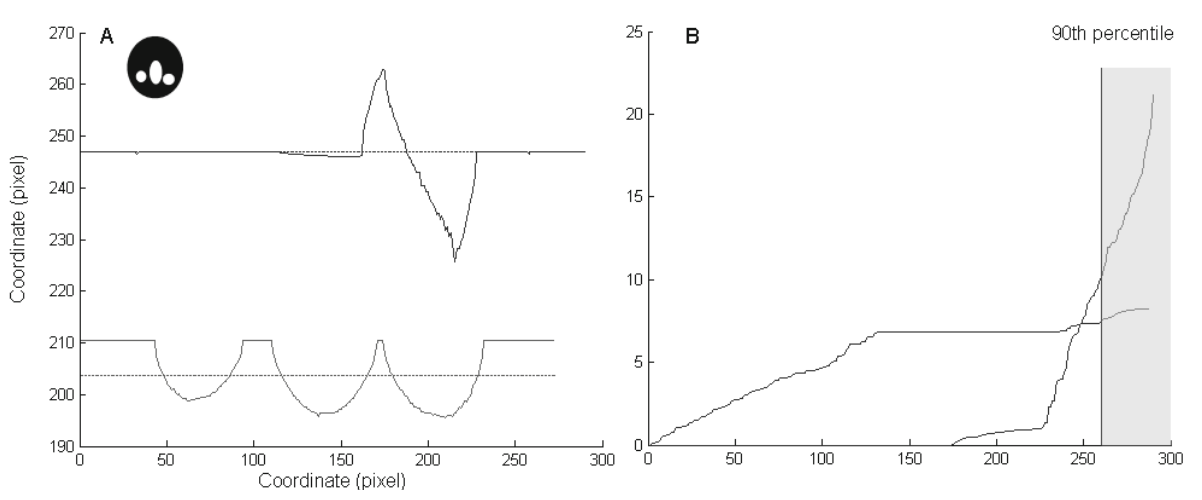

Figure 2. Median-based pupil center detection method. The algorithm was tested on a pupil presenting three marks of corneal reflection (A). The top curve represents the barycentres of the vertical vectors constituting the pupil have been plotted as a function of their coordinates in abscissa (top curve). The bottom curve represents the barycentres of the horizontal vectors as a function of the coordinates in ordinates. It is obvious that the center of vector passing through the noise is shifted in the opposite direction to the noise. The respective median of these two curves is represented by the broken lines. Each median was then subtracted to their respective curve and the resulting absolute values were increasingly sorted $(B)$. The gray zone represents the filtered values $(10 \%$ of the maximum values). This method was carried out five times in order to obtain a stabilized median, and thus an accurate estimation of the pupil center coordinates.

outer diameter is determined by the sclera. However, video formatting can sometimes distort images. For example, a circle such as the pupil can be transformed into an ellipse because of flattening. Therefore, the transformation of the iris from Cartesian to polar coordinates needs the vertical and horizontal radii of the pupil to be taken into account otherwise the coordinate transformation would be biased by this noncircularity. To avoid such a bias, we calculated the ratio $K$ between the two pupil-ellipse radii $\left(K=r_{\text {phoriz }}\right.$
( $\left.r_{\text {pvert }}\right)$, each ellipse being computed with the use of an algorithm derived from the above mentioned papers describing the classical ellipse fitting method. This ratio was used to calculate the two radii of the outer ellipse of the iris. Then the iris was transformed into polar coordinates as described in Figure 3. Elements of the selected iris region of the image I (Figure $3 \mathrm{~A}$ ) were projected into a new matrix $\mathrm{P}$ of polar coordinates denoted $(r, \alpha)$ (Figure 3B) defined from the pupil center coordinates $\left(x_{\text {pup }}, y_{\text {pup }}\right)$.
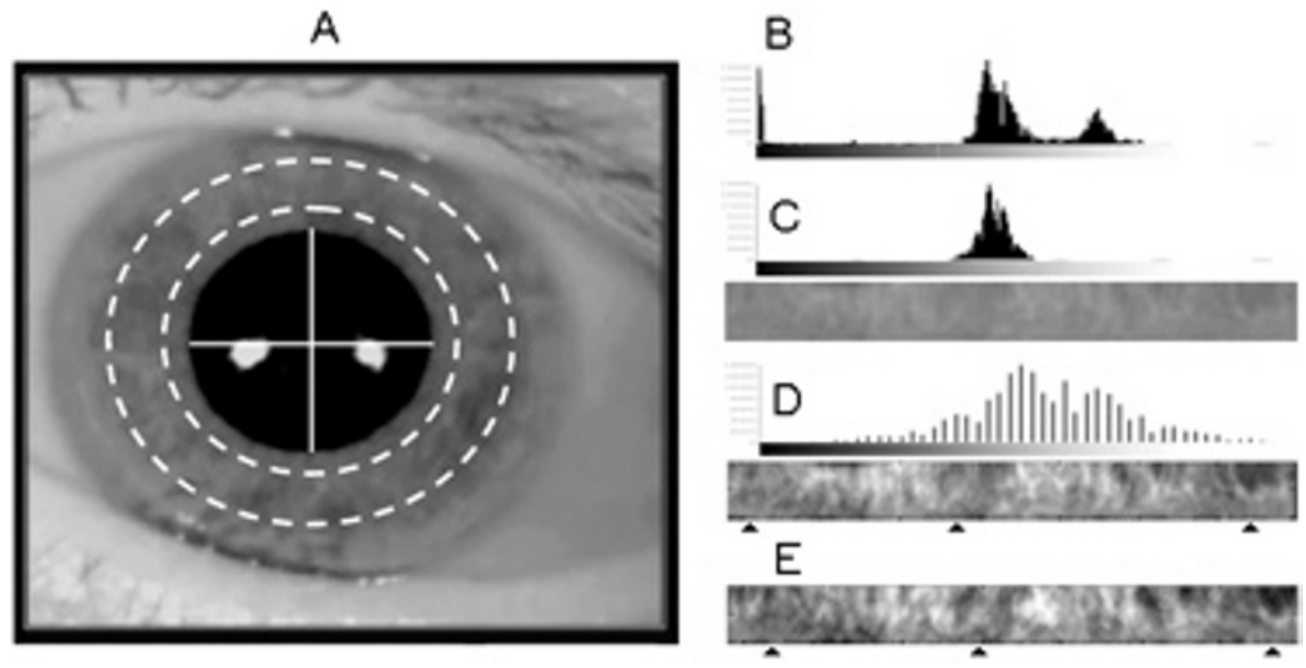

Figure 3. The algorithm deducts a pupil-centered iris zone to be analyzed, neglecting the corneal reflection of infrared light. A human-machine interface allows definition of a maximum iris annulus zone with various possible length and width according to subjects and conditions (A). The iris structure generally reveals histogram ranging from 90 to 150 whereas the luminous intensity of the pupil is usually restricted to the five lowest values of the intensity histogram, allowing easy pupil detection (B). The iris annulus is then converted into polar coordinates (C) and its histogram on the 256 gray levels spectrum is defined (D). In order to improve the contrast of the iris annulus, the gray values were stretched such that they overlap the whole spectrum. An illustration of a few degrees of torsion on the iris annulus during head tilt is provided (E). 
$r$ defines the radius and $\alpha$ defines the azimuth. The polar transformation is described thus:

$$
\mathrm{P}(r, \alpha)=\mathrm{I}\left[x_{\text {pup }}+r_{\text {ihoriz }} \cdot \cos (\alpha), y_{\text {pup }}+r_{\text {ivert }} \cdot \sin (\alpha)\right] \text {. (1) }
$$

Note that $r_{\text {ihoriz }}$ and $r_{\text {ivert }}$ represent the horizontal and vertical widths of the iris annulus, respectively. If the image I is not distorted, the iris, as the pupil, constitutes a perfect circle. In this case, $r_{\text {ihoriz }}$ and $r_{\text {ivert }}$ are equal. By contrast, if the image is distorted, the ratio $\mathrm{K}$ is also used to calculate the width and the height of the iris annulus $\left(r_{\text {ihoriz }}=\right.$ $\left.K \cdot r_{\text {ivert }}\right)$.

Classically, "iral arcs" are used (i.e., vectors of 256 pixels which spanned $70^{\circ}$ ) (e.g., Moore et al., 1996). We opted for adjustable iral arcs in order to use the largest exploitable iris zone. The experimenters selected the most reliable iral zone - that is, the most contrasted one (large or small number of vectors or degrees).

One major drawback with the classical polar crosscorrelation method is the necessity to maintain a small and constant pupil diameter using pilocarpine. Indeed, without this precaution, the pupil size tends to change as a function of time. Thus, the iral arcs of the current image do not correspond any more to the iral arcs of the reference image, which leads to errors of a couple of degrees in the calculation of ocular torsion. To avoid such a bias without using pharmacology to maintain constant pupil diameter (i.e., to avoid the associated constraints and undesirable side effects), we used the elastic pupil-iris model that relies on a proportional transformation principle (Zhu et al., 2004), as described by

$$
r_{\mathrm{arc}}^{\prime}=r_{\mathrm{p}}^{\prime}+\frac{r_{\mathrm{arc}}-r_{\mathrm{p}}}{r_{\mathrm{i}}-r_{\mathrm{p}}}\left(r_{\mathrm{i}}-r_{\mathrm{p}}^{\prime}\right),
$$

with $r_{\text {arc }}^{\prime}$ representing the radius of the iral arc of the current image, $r_{\mathrm{p}}$ and $r_{\mathrm{p}}^{\prime}$ the radii of the pupil of the reference image and current image, respectively, $r_{\mathrm{i}}$ the radius of the iris (which remains constant whatever the pupil size), and $r_{\mathrm{p}}^{\prime}$ the radius of the pupil of the current image. Each iral zone was then cross-correlated with its respective zone on the reference image. Despite a longer processing time, this method provides main advantages leading to a greater accuracy of the cross-correlation algorithm: (1) the reduction of eye-eccentricity-induced errors and (2) the avoidance of noisy zones (e.g., eye-blinks, corneal reflection).

Luminous intensity normalization of the image. To address variation in luminous intensity of images in the same sequence, we used an algorithm which stretches the gray values of the iris matrix. The first step is to localize, from the image histogram, the gray levels corresponding to the iris zone. The second step consists of stretching the gray level such that the lowest gray value of the iris corresponds to zero and the highest one to 255 (normalization in magnitude), as described in Figures 3B and 3C.

Cross correlation. In order to calculate the degree of torsion, we need to compare the iris matrix at time $t$ with the reference iris matrix. More precisely, each iris matrix $(n \times m$ matrix $)$ is constituted by one or more iris vectors $(n \times 1$ matrix $) .{ }^{1}$
The cross-correlation between two functions $f$ and $g$ is defined by

$$
f \times g \equiv f(t) * \bar{g}(-\mathrm{t}),
$$

where the asterisk is the product of convolution and $\bar{g}(t)$ the conjugate of $g(t)$.

The cross-correlation also corresponds to

$$
f \times g \equiv \mathrm{FT}^{-1}\{\mathrm{FT}[f] \cdot \mathrm{FT}[\bar{g}]\},
$$

where the FT indicates the fast Fourier transform.

The cross-correlation coefficient $r$ was calculated as in (5), using the $x$ corr MATLAB function. In MATLAB, the coefficient of correlation is not normalized, and the integral is approximated by means of the sum of elements:

$$
r_{f f_{\text {ref }}}(d)=f \times f_{\text {ref }}=\int_{-\infty}^{+\infty} f(x+d) \cdot \overline{f_{\text {ref }}}(x) d x,
$$

where $f_{\text {ref }}$ is the reference iris vector and $f$ is another iris vector at different angular offsets $d$. The coefficient of correlation varies as a function of $d$. The angular offset at the correlation peak is proportional to eye torsion. The maximum torsional movement of the eye is limited to $20^{\circ}$ (Zhu et al., 2004). This value was used as the maximum angular offset for the cross-correlation to reduce the time of analysis. Each iral vector provided an angular offset. The median of these values was used since it is the most reliable angular offset. Indeed, pictures can sometimes include some noise essentially due to corneal reflection which remains fixed while the eye is rotating. Since a large iris region of the image is exploited, this noise can easily be suppressed by using the median, whereas average measures would automatically lead to errors in torsion measurement. To convert this value into degrees, the raw offset value is multiplied by the default resolution of the vector $\left(0.25^{\circ}\right.$; however, this resolution can be modified by the experimenter by means of the human-machine interface if necessary).

This classical method is known to be reliable when the eye is centered in the orbit (Clarke, Teiwes, \& Scherer, 1991; Hatamian \& Anderson, 1983; Moore et al., 1991; Vieville \& Masse, 1987). However, the accuracy decreases with eye eccentricity (Haslwanter \& Moore, 1995). If the experimental task requires the eyes to move more than $10^{\circ}$, this drift can be corrected by taking into account the geometrical properties of the eye (3-D spherical model of the eye) (Moore et al., 1996). Such a correction allows accurate measurement of ocular torsion for eye eccentricity up to $30^{\circ}$ instead of $10^{\circ}$.

Head tracking. The Eyelink II headband is equipped with a head tracking infrared camera assigned to film four infrared LEDs. Generally, these markers are disposed on the four corners of the screen placed in front of the subject. Head orientation in space is then inferred from the markers positions. The markers positions in space are displayed on the host computer screen as four spots in a small window (Figure 1), and are available on the video recording. The goal is to detect the four markers, to track them, and to calculate head orientation.

The first step for head tracking is the detection of the four markers. This is carried out by transforming the gray- 
scale image into a black-and-white binary image. The markers detection has been optimized for a threshold of 10 out of 256 gray levels. For the first image of the sequence, the experimenter has to click on each marker in a specific order. Then, the algorithm calculates the "center of mass" of each marker by attributing one unit of mass to each pixel constituting the marker. As mentioned previously, such a method is classically used to detect the pupil center. However, we thought that it was more reliable to calculate the center of mass of the marker than the center of mass of the pupil since there is no noise such as infrared light reflection on the former.

Then the horizontal and vertical orientations of the set of markers are calculated (mean slope between the two vertical segments, mean slope of the two horizontal segments) and converted to degrees. Again, if the image is flattened, the deformation can be compensated by multiplying the between-markers distance (for the width or height) by a ratio previously calculated from the actual distance between the four infrared LEDs.

The tracking algorithm consists in using the center of mass of the marker of the previous frame to test the "binarity" of the adjacent matrix elements and then to calculate the new center of mass of the marker on the current frame. This algorithm allows the fast calculation of head orientation in space, since head movement is opposite to that of the markers.

\section{Validation}

Pupil center detection. We tested our pupil center detection algorithm on computer-generated binary im- ages with noise such as corneal reflection, which is often observed on actual video sequence. Figure 4 compares three methods of pupil detection: (1) the classical center of mass method, (2) a method that consists in suppressing the noise, ${ }^{2}(3)$ the algorithm we have presented previously (median-based). Results of our validation tests confirm that the center of mass based algorithm leads to pupil center estimation errors whenever the pupil presents asymmetrical noise. Figure 4 also shows there are residual errors of relatively small amplitude when using a center of mass-based algorithm with noise correction. However, it has been reported that even a small error of 1 or 2 pixels in determining the pupil center can induce torsion calculation errors up to a couple of degrees, for all algorithms used to measure torsion (Hatamian \& Anderson, 1983; Sung \& Reschke, 1997). Finally, our method seems to provide the most interesting results. Although parts of the pupil were occluded, the center was accurately detected for all conditions with no resulting radial error.

Polar cross-correlation. Although the accuracy of the classical polar cross correlation method has already been validated in the literature (Hatamian \& Anderson, 1983), we wanted to test the validity of our specific algorithm. To reach this goal, we developed an ocular torsion simulation based on controlled rotation of a virtual eye and a head rotation simulation based on the controlled virtual displacement of an image of the four virtual markers. We generated two video sequences using MATLAB. The first one was the rotation of an eye picture (image size: $260 \times 251$ ) which mimicked slow and fast movements as shown in Figure 5.

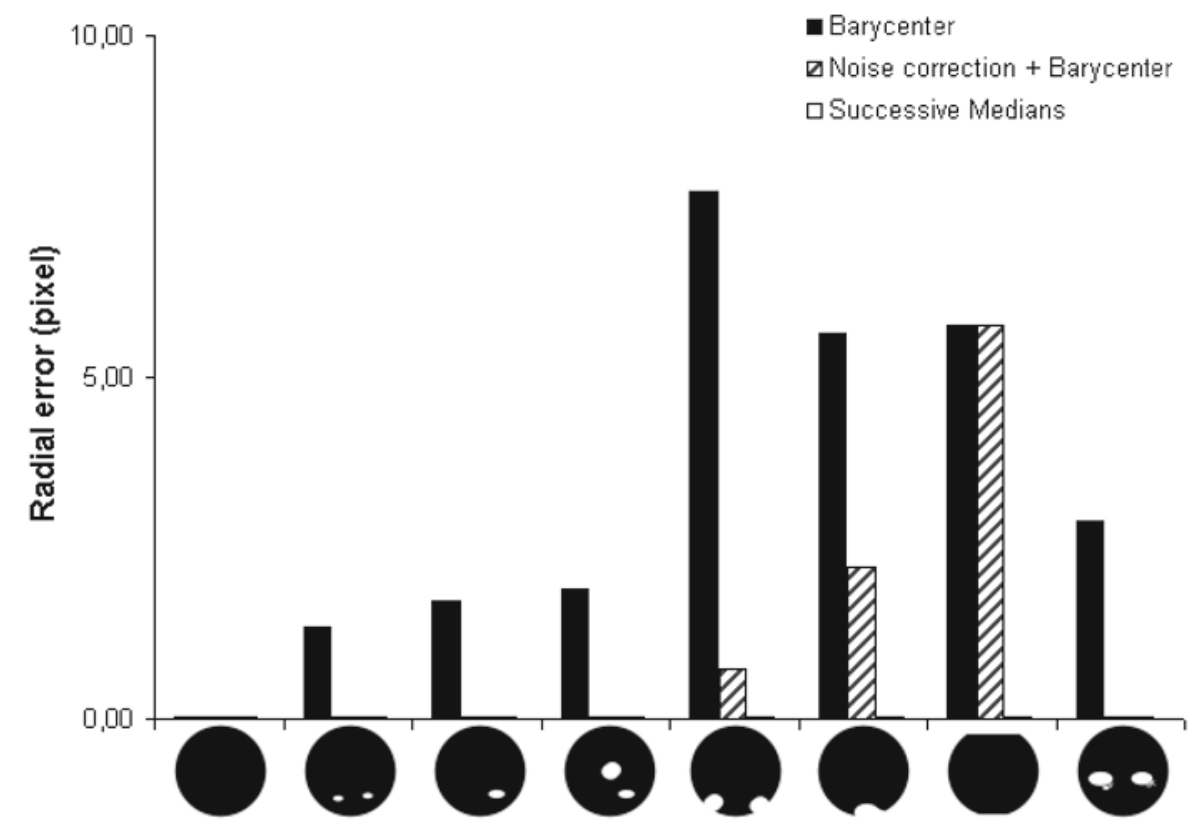

Figure 4. Illustration of the validation of the pupil detection method. Radial error in the detection of the pupil center was plotted as a function of tested images (computer generated binary images [200 $\times 200$ pixels] which were inspired from real images). Three methods were tested: (1) the classical center of mass method (black bars), (2) the center of mass method after noise correction (striped bars, see details in foot notes for details of the noise correction method), (3) our medianbased method (white bars). 


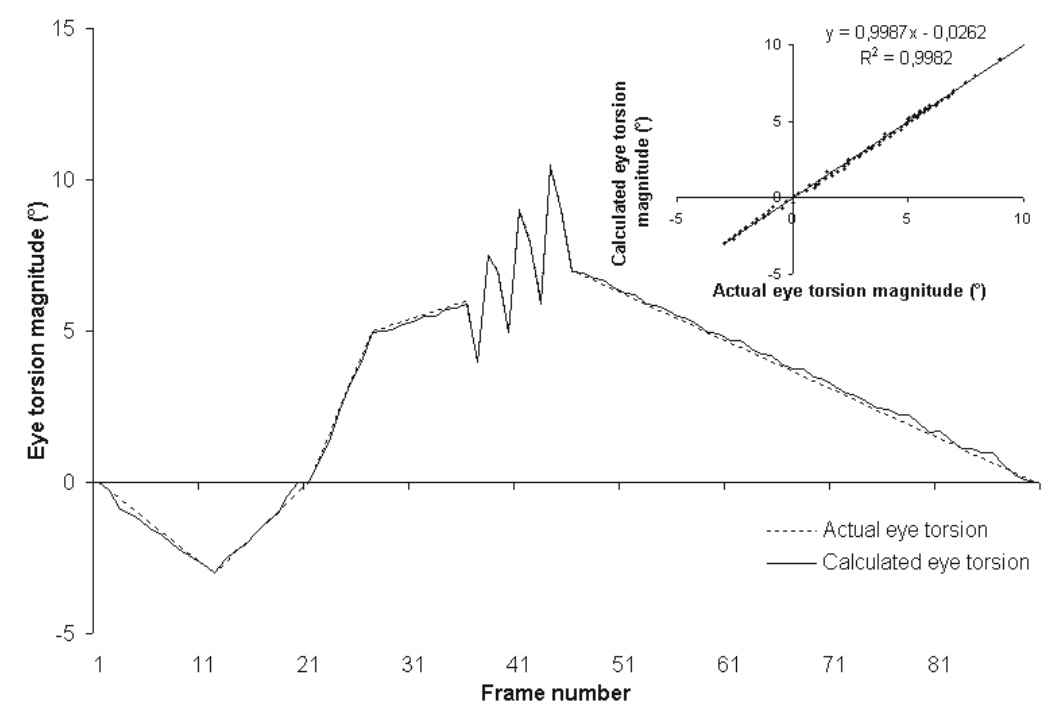

Figure 5. Results of the validation test of the polar cross-correlation method based on the simulated eye torsion (computer-generated eye images). The main panel of the figure presents the orientation of both the actual (dotted line) and the calculated (full line) orientations of the virtual eye as a function of the frame number. In the small panel, the calculated orientation is plotted as a function of the actual orientation.

The second one was the rotation of a group of 4 dots randomly disposed on an image (image size: $160 \times 151$ pixels) as shown in Figure 6. Our method was tested on these two image sequences. Figure 5 shows the eye orientation calculated by the polar cross-correlation method and the eye actual orientation. The coefficient of correlation between these two variables is close to 1 . Moreover, the slope of the regression line is also close to 1 and the intercept close to 0 . On the basis of these results, the method appears to be highly reliable. Figure 6 shows the markers orientation calculated with our "head tracking" algorithm as well as their actual orientation. The shift observed in the orientation of the group of markers is due to their initial disposition which was voluntarily randomized to test the robustness of our algorithm. Such a shift between the calculated and the actual orientation can be canceled by a simple data "rebase" (calibration). Here again, the method appears to be reliable.

Compensation for pupil size changes. From a part of the computer-generated image sequence, we mimicked pupil dilatation (MATLAB homothetic transformation: $82 \%-110 \%$ of the original size) in presence or in absence of ocular torsion and compared the torsion measurement of our specific algorithm with that of the classical cross-correlation method. We observed that the classical algorithm which does not take the pupil size changes into account led to important biases. According to Figure 7, it appears necessary to take the pupil size changes into account since pupil dilatation without any torsion can be interpreted as ocular torsion up to $1^{\circ}$ with the classical method. Note that without taking into account pupil size changes, we

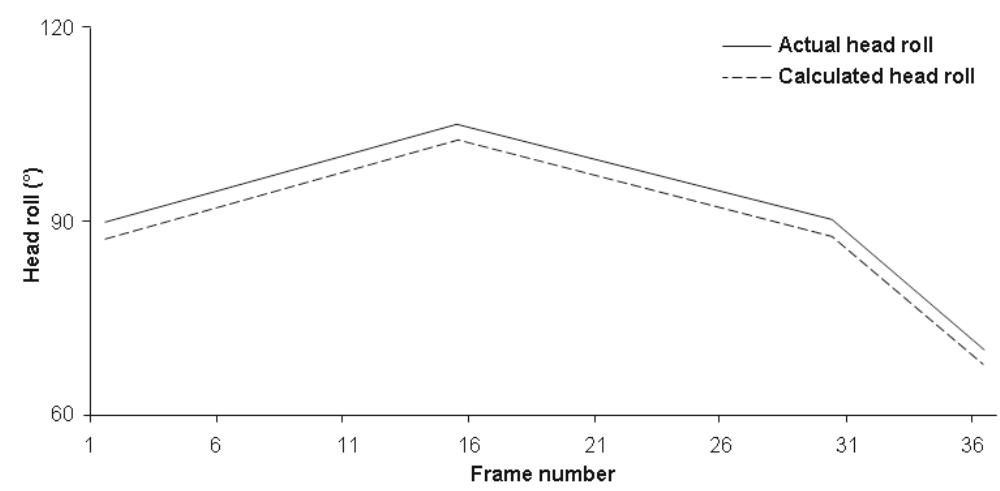

Figure 6. Results of the validation test of the marker tracking method on the computer-generated images. Orientation of both the actual (full line) and calculated (dotted line) markers are represented as a function of the frame number. The shift between the calculated and the actual orientation (see text for explanation) can be canceled by a simple data rebase. 


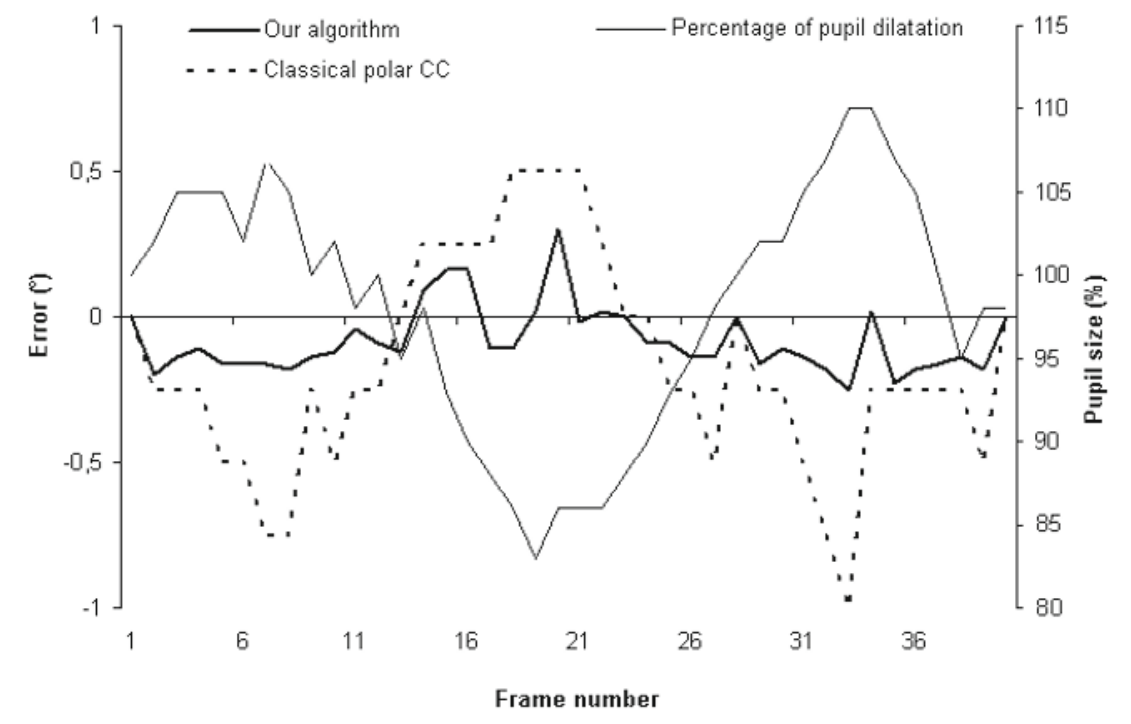

Figure 7. Validation of the taking into account of the pupil size changes. The pupil of computer-generated images was controlled (thin full line). Pupil diameter changes are expressed in percent of its initial size (right ordinate axis). These modifications in pupil size lead to a compression or an expansion of the iris, modifying the selected iris zone if the compensation algorithm is not integrated. Torsion was measured on a video sequence that presented fast and slow eye torsion up to $10^{\circ}$. The error in torsion measurement (left ordinate axis) was calculated with (bold full line) and without the compensation (dotted line) the pupil size changes. Note that the error is significantly correlated to the pupil size with both algorithms (without compensation: $y=-4.1+3.86, r^{2}=.68$; with compensation: $y=-0.88+0.78, r^{2}=.32$ ). However, the correlation remains greater without the compensation algorithm.

observed a significant correlation between the error in torsion measurement and the pupil size (regression line: $\left.y=-0.165 x+0.9534 ; r^{2}=-.67 ; p<.001\right)$. This correlation suggests that the error may be even larger with greater modifications in pupil size. With our algorithm, the absolute error remains below $0.25^{\circ}$ (theoretical accuracy of the polar cross-correlation method) and can even be attenuated by filtering these data.

Behavioral illustration. To further illustrate our method, our algorithm was also tested on a human subject during head roll tilt (Figure 8). As the subject tilted his head leftward, the eye counterrolled. Both the phasic component, a kind of vestibular torsional nystagmus involving both semicircular canals and otolith signals generated during head tilt, and the tonic component, the nonzero torsion plateau mainly due to otolith signal caused by head tilt, of eye torsion are easily observable and distinguishable. In this specific condition, the final magnitude of the eye countertorsion reached circa $10 \%$ of head tilt, as predicted in the literature (e.g., Collewijn et al., 1985).

Limits. Although video-based systems constitute interesting solutions to record eye movements, the methods classically used may present some problems that still remain unsolved. For instance, the low sampling rate constitutes one of the most evoked drawbacks (van der Geest \& Frens, 2002). This criticism especially concerns 2-D eye movements and more precisely saccadic eye movements which are the fastest movements humans can produce (up to $800^{\circ} / \mathrm{sec}$ ). However, the limitation vanishes when study- ing ocular torsion since the velocity is much lower (mean torsional velocity rarely exceeds $5^{\circ} / \mathrm{sec}$ ) (Kori, SchmidPriscoveanu, \& Straumann, 2001). Accordingly, a $30 \mathrm{~Hz}$ sampling rate is sufficient to obtain reliable data (most studies use $30 \mathrm{~Hz}$ or less).

Unfortunately with our method, the classical potentialities of Eyelink II such as online providing 2-D eye movements are not available anymore because the EyeLink II system must be run displaying the "camera setup screen." This mode must be used in order to have both a large image of the eye (i.e., the best resolution for the video capture) and information about head markers. Eye position recording and Eyelink basic functions are not available in this mode. However, it is possible to access these values with an offline analysis and the use of an appropriate calibration. The horizontal and vertical eye movements must be limited since the polar cross-correlation method leads to torsional errors at eccentric eye positions. The difficulty to distinguish translation from rotation constitutes the main limit of all existing methods. Even artificial neural networks (e.g., Guillemant, Ulmer, \& Freyss, 1995) cannot take into account eye eccentricity-induced distortion of the image. Therefore, for large eccentricities, we suggest to implement the useful and complementary 3-D spherical model (Moore et al., 1996). Nevertheless, if the interaction between lateral and torsional eye movements is not the issue under scrutiny, we simply suggest including a fixation target in the display to prevent from eccentric saccades during torsion measurement. 

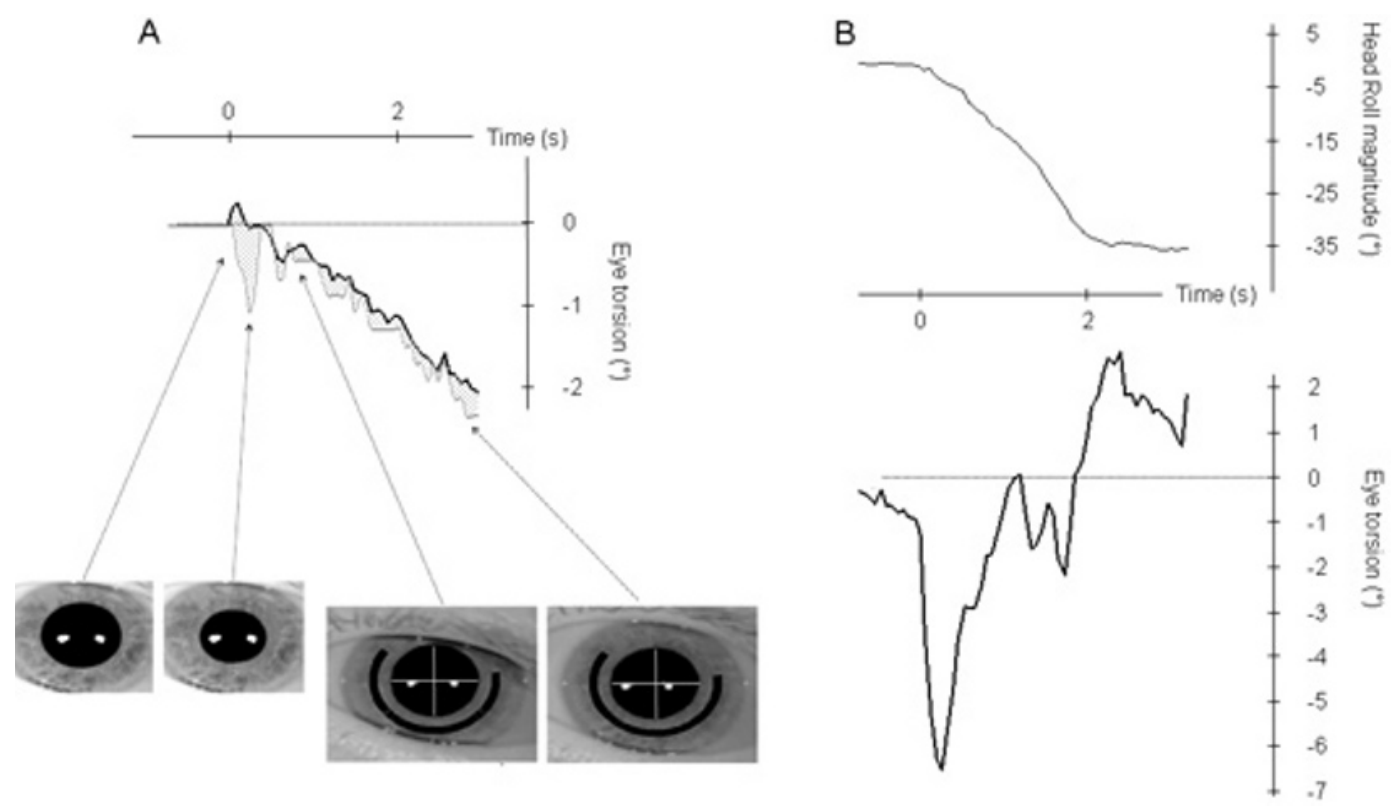

Figure 8. (A) Illustration of torsion measures on a real human subject performed with (black line) and without (grey line) our algorithms. Two typical conditions leading to clear differences between the classical crosscorrelation method and our adapted method are presented: a case of pupil dilatation together with a case of moving corneal reflection. (B) Illustration of the ocular counterroll during head tilt. Head and eye roll were measured during a counterclockwise head tilt (circa $30^{\circ}$ ). Phasic (vestibular nystagmus) and tonic components of the ocular counterroll can easily be observed. Signals are not filtered.

\section{CONCLUSION}

Here we show that the Eyelink II device (or any similar equipment) can be used to record ocular torsion. More generally, we propose a method for torsion analysis which is applicable to all other video-based recordings. The main interest of this method is a greater precision for torsion measurement which benefits from: (1) the use of large iris regions, (2) the development of an original, straightforward and efficient median-based procedure to detect pupil center, and (3) accounting for pupil size changes. In addition, since pilocarpine hydrochloride is not used when using this method, its associated drawbacks vanish and medical supervision is not necessary. This may provide psychologists and neurophysiologists with an adaptive and efficient tool to study this particular ocular movement that remains poorly understood, but is a key element in the insight of sensorimotor integration.

\section{AUTHOR NOTE}

This work was supported by Grant ACI 6042 from the French Ministry of Research and a grant from the MSHS, XIIème CPER, University of Poitiers. The authors are grateful to David Chesnet for his help with the use of Eyelink II, Sabrina Lee for carefully reading and correcting the manuscript, and two anonymous reviewers for their helpful comments and suggestions. Correspondence concerning this article should be addressed to P. Boulinguez, Laboratoire Performance, Motricité, Cognition, Maison des Sciences de l'Homme et de la Société, 99 av. du Recteur Pineau, F-86000 Poitiers, France (e-mail: pbouling@univ-poitiers.fr).

\section{REFERENCES}

Bockisch, C., \& Halswanter, T. (2001). Three-dimensional eye po- sition during static roll and pitch in humans, Vision Research, 41, 2127-2137.

Clarke, A. H., Ditterich, J., Drüen, K., Schönfeld, U., \& SteinEKE, C. (2002). Using high frame rate CMOS sensors for three-dimensional eye tracking. Behavior Research Methods, Instruments, \& Computers, 34, 549-560.

Clarke, A. H., Teiwes, W., \& Scherer, H. (1991). Video-oculography: an alternative method for measurement of three dimensional eye movements. In R. Schmidt \& D. Zambarbieri (Eds.), Oculomotor control and cognitive processes (pp. 431-443), Amsterdam: Elsevier.

Collewijn, H., Van der Steen, J., Ferman, L., \& Jansen, T. C. (1985). Human ocular counterroll: Assessment of static and dynamic properties from electromagnetic scleral coil recordings, Experimental Brain Research, 59, 185-196.

Curthoys, I. S., \& Wade, S. W. (1995). Ocular torsion position and the perception of visual orientation. Acta Otolaryngologica Supplementum, 520(Pt. 2), 298-300.

Diamond, S. G., \& Markham, C. H. (1981). Binocular counterrolling in humans with unilateral labyrinthectomy and in normal controls. Annals of the New York Academy of Sciences, 374, 69-79.

DonDERs, F. C. (1848). Beitrag zur Lehre von den Bewegungen des menschlichen Auges. Hollandische Beitreig zur Anatomie \& Physiologie Wissenschaften, 1, 105-145.

Finke, R. A., \& HeLd, R. (1978). State reversals of optically induced tilt and torsional eye movements. Perception \& Psychophysics, 23, 337-340.

Groen, E., Bos, J. E., Nacken, P. F. M., \& De Graaf, B. (1996). Determination of ocular torsion by means of automatic pattern recognition. IEEE Transactions on Biomedical Engineering, 43, 471-479.

Guillemant, P., Ulmer, E., \& Freyss, G. (1995). 3-D eye movement measurements on four Comex's drivers using video CCD cameras, during high pressure driving. Acta Otolaryngologica Supplementum, 520(Pt. 2), 288-292.

HALL, R. (1983). Image processing algorithms for eye movement monitoring. Computers \& Biomedical Research, 16, 563-579.

Haslwanter, T., \& Moore, S. T. (1995). A theoretical analysis for three dimensional eye position measurement using polar cross correlation. IEEE Transactions on Biomedical Engineering, 42, 1053-1061. 
Hatamian, M., \& Anderson, D. J. (1983). Design considerations for a real-time ocular counterroll instrument. IEEE Transactions on Biomedical Engineering, 30, 278-288.

Irie, K., Wilson, B. A., Jones, R. D., Bones P. J., \& Anderson T. J. (2002). A laser-based eye-tracking system. Behavior Research Methods, Instruments, \& Computers, 34, 561-572.

JAMPEL, R. S., \& SHI, D. X. (2002). The absence of so-called compensatory ocular countertorsion, the response of the eyes to head tilt. $\mathrm{Ar}$ chives of Ophthalmology, 120, 1331-1340.

Kori, A. A., Schmid-Priscoveanu, A., \& Straumann, D. (2001). Vertical divergence and counterroll eye movements evoked by wholebody position steps about the roll axis of the head in humans. Journal of Neurophysiology, 85, 671-678.

Kushner, B. J. (2004). Ocular torsion: rotation around the "why" axis Journal of American Association in Pediatrics \& Ophthalmologic Strabismus, 8, 1-12.

Moore, S. T., Curthoys, I. S., \& McCoy, S. G. (1991). VTM: An image-processing system for measuring ocular torsion. Computer Methods \& Programs in Biomedicine, 35, 219-230.

Moore, S. T., Haslwanter, T., Curthoys, I. S., \& Smith, S. T. (1996). A geometric basis for measurement of three-dimensional eye position using image processing. Vision Research, 36, 445-459.

NaKayama, K. (1974). Photographic determination of the rotational state of the eye using matrices. American Journal of Optometry \& Physiological Optics, 51, 736-742.

Онм, J. (1928). Die Hebelnystagmographie. Gradfes Archive zur Ophthalmologie, 120, 235-252.

Robinson, D. (1963). A method of measuring eye movement using scleral search coil in a magnetic field. IEEE Transactions on Biomedical Engineering, 10, 137-145.

Schreiber, K., \& Haslwanter, T. (2004). Improving calibration of 3 -D video oculography systems. IEEE Transactions on Biomedical Engineering, 51, 676-679.
Sung, K., \& Anderson, D. J (1991). Analysis of two video eye tracking algorythms. Proceedings of the Annual International Conference IEEE Engineering in Medicine \& Biology Society, 13, 1949-1950.

SunG, K., \& ReschKe, M. F. (1997). A model-based approach for the measurement of eye movements using image processing. NASA technical paper, 3680, 1-39.

VAn der Geest, J. N., \& Frens, M. A. (2002). Recording eye movements with video-oculography and sceral search coils: a direct comparison of two methods. Journal of Neuroscience Methods, 114, 185-195.

Vieville, T., \& MASsE, D. (1987). Ocular counter-rolling during active head tilting in humans. Acta Otolaryngologica, 103, 280-290.

Wade, S. W., \& CurThoys, I. S. (1997). The effect of ocular torsional position on perception of the roll-tilt of visual stimuli. Vision Research, 37, 1071-1078.

Yamanobe, S., Taira, S., Morizono, T., Yagi, T., \& Kamio, T. (1990). Eye movement analysis system using computerized image recognition. Archives of Otolaryngology, 116, 338-341.

Zhu, D., Moore, S. T., \& RaPhan, T. (1999). Robust pupil center detection using a curvature algorithm. Computer Methods \& Programs in Biomedicine, 59, 145-157.

Zhu, D., Moore, S. T., \& Raphan, T. (2004). Robust and real-time torsional eye position calculation using a template-matching technique. Computer Methods \& Programs in Biomedicine, 74, 201-209.

\section{NOTES}

1. An iris vector is an iral arc converted into polar coordinates.

2. Fitting an ellipse to the 4 estimated borders (top, bottom, left, and right) of the pupil allows to "fill in" the noise inside the pupil in most cases, but can lead to strong errors in some other cases.

(Manuscript received January 16, 2006; revision accepted for publication July 5, 2006 .) 